und

Max-Planck-Institut für Kohlenforschung

Journal Article published in: J. Phys. Chem. Lett., 2018, 9, 17, 5116-5120, ACS Publications

https://doi.org/10.1021/acs.jpclett.8b01846

This article may be used for non-commercial purposes.

\title{
High Lithium Transference Number Electrolytes Containing Tetratriflylpropene's Lithium Salt
}

\author{
J. Popovic ${ }^{\dagger}$, D. Höfler ${ }^{\S^{\star}}$, J. P. Melchior ${ }^{\dagger}$, A. Münchingert, B. List ${ }^{\S}$, J. Maier ${ }^{\dagger}$ \\ † Max Planck Institute for Solid State Research, Stuttgart, Germany \\ $\S$ Max Planck Institute for Coal Research, Mülheim an der Ruhr, Germany \\ \$ Current address: Oak Ridge National Laboratory, Neutron Scattering Division, Oak Ridge, TN, USA \\ Corresponding authors: Jelena Popovic, popovic@fkf.mpg.de; Denis Höfler, hoefler@kofo.mpg.de
}

KEYWORDS lithium electrolyte, ionic conductivity, ion pairing, superacid, C-H acid

\begin{abstract}
Electrolytes with a high lithium transference number linked with high ionic conductivity are urgently needed for high power battery operation. In this work, we present newly synthesized lithium tetra(trifluoromethanesulfonyl)propene used as a salt in glyme-based salt-in-solvent electrolyte. We employ impedance spectroscopy in symmetric Li/electrolyte /Li cells and pulsed field gradient nuclear magnetic resonance spectroscopy to investigate the lithium conduction mechanism. We find predominant lithium conductivity with very high lithium transference numbers ( $70 \%$ from the polarization experiments) and three times higher ionic conductivity compared to well-known lithium triflate in diglyme electrolyte. This is a consequence of the reduced mobilities of large anions linked with improved ionic dissociation due to the lower basicity.
\end{abstract}

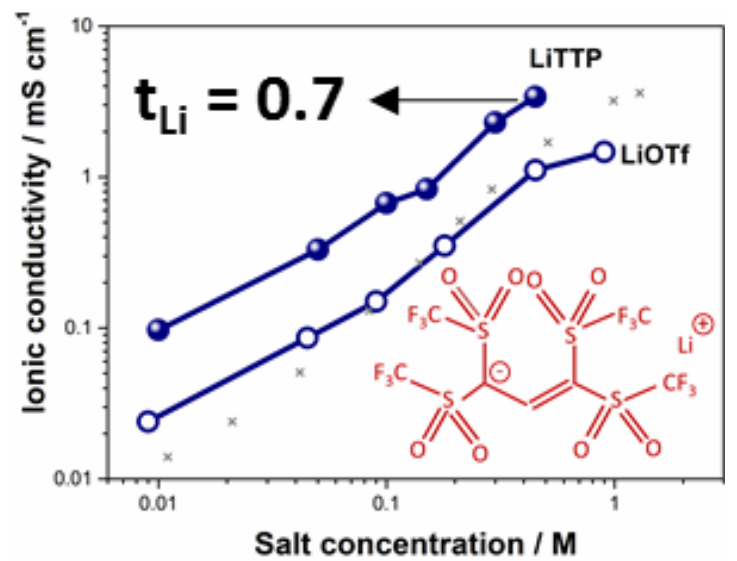


Lithium salts are a key component of lithium batteries providing the ionic species needed for charging/discharging processes. Their properties are decisive for the ionic conductivity, lithium transference number, solid electrolyte interphase (SEI) formation, electrochemical window, and thermal stability of the practically used non-aqueous electrolytes. ${ }^{1-3}$ One of the most common negative aspects of the standard non-aqueous liquid electrolytes linked with the salts such as lithium hexafluoroarsenate $\left(\mathrm{LiAsF}_{6}\right)$, lithium tetrafluoroborate $\left(\mathrm{LiBF}_{4}\right)$, lithium perchlorate $\left(\mathrm{LiClO}_{4}\right)$, lithium trifluoromethanesulfonate $\left(\mathrm{LiCF}_{3} \mathrm{SO}_{3}, \mathrm{LiOTf}\right)$, and the presently prevailing lithium hexafluorophosphate (LiPF 6 ), is their relatively low lithium transference number, which leads to concentration polarization, detrimental for high power battery operation. Anions such as $\mathrm{BF}_{4}^{-}, \mathrm{ClO}_{4}{ }^{-}, \mathrm{CF}_{3} \mathrm{SO}_{3}{ }^{-}$, and $\mathrm{PF}_{6}{ }^{-}$have smaller solvation shells consisting of one to two solvent molecules when compared to the distinctly smaller $\mathrm{Li}^{+}$ion, binding four to six solvent molecules. As a consequence, anions are fairly mobile in typical solvents at salt concentrations, $c_{\text {salt, }}$ around $1 \mathrm{M}$. $^{3}$ Additionally, the often high degree of ionic association in such electrolytes, enabled through superimposition of Coulombic and short range interactions with formation of effectively neutral species, plays a critical role in lowering the values of total ionic conductivity, $\sigma_{\mathrm{AC}}$.

Recently, efforts have been made to unravel the complex molecular structures of the conventional carbonate lithium electrolytes. ${ }^{4-8}$ Advancement has also been achieved in the prediction ${ }^{9,10}$ or preparation of diverse electrolytes with larger and more charge delocalized anions potentially suppressing ionic association. Suggested molecules include bis(trifluoromethane)sulfonimide $\left(\left[\left(\mathrm{CF}_{3} \mathrm{SO}_{2}\right){ }_{2} \mathrm{~N}\right]^{-}\right.$, TFSI), ${ }^{11}$ bis(pentafluoroethylsulfonyl)imide $\left(\left[\left(\mathrm{SO}_{2} \mathrm{~F}\right)_{2} \mathrm{~N}\right]^{-}, \mathrm{FSI}\right),{ }^{12}$ bis(oxalato)borate $\left(\left[\left(\mathrm{C}_{2} \mathrm{O}_{4}\right)_{2} \mathrm{~B}\right]^{-}, \quad \mathrm{BOB}\right),{ }_{13}$ (malonatooxalato)borate $\left(\left[\left(\mathrm{OCH}_{3}\right)_{4} \mathrm{~B}\right]^{-}, \mathrm{MOB}\right),{ }^{14} \quad 4,5-\mathrm{dicyano}-2-$ (trifluoromethyl)imidazole $\left(\left[\mathrm{C}_{6} \mathrm{~F}_{3} \mathrm{~N}_{4}\right]^{-}\right.$, TDI), ${ }^{15,16}$ fluoromalonatoborate ${ }^{17,18}$, anilinyl-perfluorosulfonamide ${ }^{19}$, and fluorinated alkoxyaluminates ${ }^{20}$. Unfortunately, even when the total ionic conductivities of the above-mentioned electrolytes are satisfactory, polarization in a Li/electrolyte/Li cell and/or pulsed field gradient nuclear magnetic resonance (PFG NMR) experiments reveal a predominant anionic conductivity mechanism.

1,1,3,3 Tetra(trifluoromethanesulfonyl)propene (TTP) is a newly synthesized, highly acidic C-H acid. After deprotonation, the generated negative charge can be delocalized over four strongly electron withdrawing $\mathrm{CF}_{3} \mathrm{SO}_{2}$ (=trifluoromethanesulfonyl=triflyl=Tf) groups. ${ }^{21}$ The high anionic stability, achieved via mesomeric and inductive properties of the four triflyl groups, minimizes its Lewis basicity and increases the Brønsted acidity of the free acid. Besides the observed high catalytic activitiy, a $\mathrm{p} K_{\mathrm{a}}$ value of -15.4 (in 1, 2-dichloroethane), ${ }^{29} \mathrm{Si} \mathrm{NMRS}$ shift experiments, and theoretical calculations confirmed its proposed high acidity. ${ }^{21}$

In this letter, we report for the first time the synthesis and electrochemical properties of 'salt-in-solvent' electrolytes containing the lithiated version of tetratriflylpropene (LiTTP, $M \mathrm{w}=576 \mathrm{~g} \mathrm{~mol}^{-1}$, Figure 1).

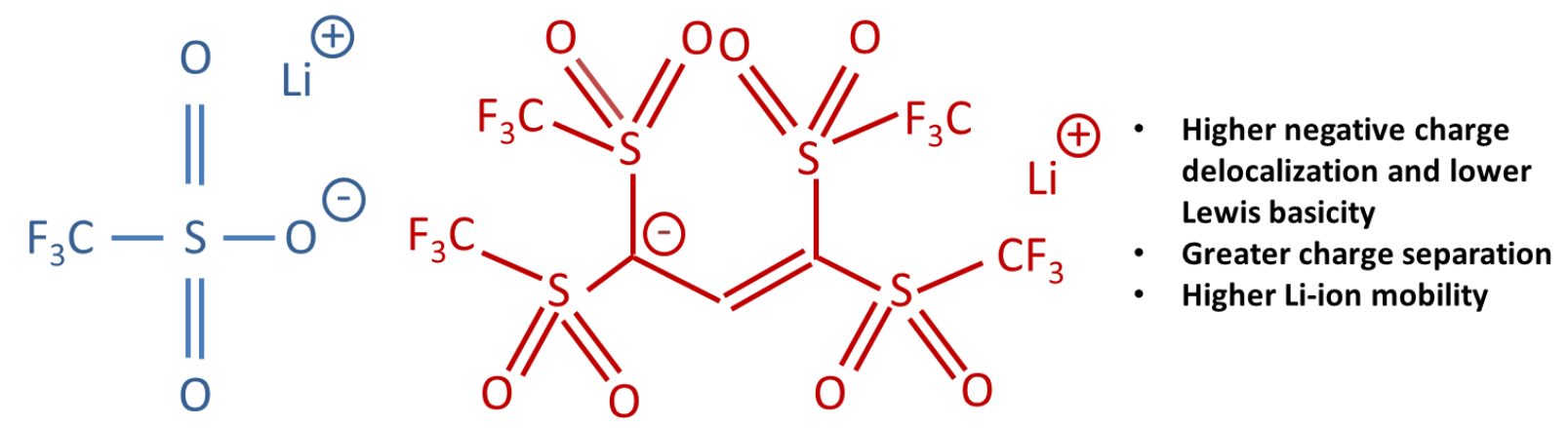

LiOTf

LiTTP

Figure 1. Comparison of LiOTf and LiTTP molecular structures. Bullet points indicate the potential beneficial properties of LiTTP in 'salt-in-solvent' electrolytes.

LiTTP can be directly prepared from TTP after treatment with $\mathrm{Li}_{2} \mathrm{CO}_{3}$ (see supplementary information). We compare LiTTP with the well-studied LiOTf as a model salt. Diglyme was chosen as a low dielectric permittivity solvent to study the influence of the anion size and electron-withdrawing effects on the salt dissociation. ${ }^{22-24}$ Despite the bigger anion size of LiTTP, salt solubility was not a limitation, and we could prepare electrolytes with up to 0.45 M LiTTP.

${ }^{1} \mathrm{H},{ }^{7} \mathrm{Li}$, and ${ }^{19} \mathrm{~F}$ tracer diffusion experiments provide a non-destructive access to the average diffusion coefficients of all molecular species containing the probed nuclei. ${ }^{25-27}$ As expected, the diffusion of the solvent, characterized by $D_{\mathrm{H}}$, NMR is faster than that of the Li or F-species, as the electrolytes under study are relatively dilute and not all of the diglyme molecules are involved in solvation processes. The averaged diffusion of $\mathrm{Li}$, and $\mathrm{F}$, containing species

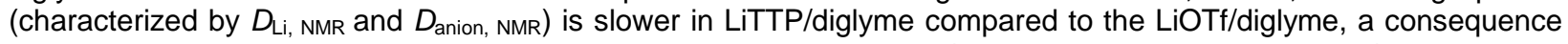
of the increased viscosity (Figure 2a). The higher viscosity of LiTTP/diglyme compared to the LiTF/diglyme is an 
indication of stronger ion/solvent interaction in LiTTP/diglyme. Peculiarly, a difference in the conduction mechanisms in diglyme electrolytes with two different salts lies in the considerably faster diffusion of Li-species compared to Fspecies $\left(D_{\mathrm{Li}, \mathrm{NMR}}>D_{\text {anion, NMR }}\right)$ in LiTTP/diglyme at all $c_{\text {salt, }}$, a strong indication of enhanced lithium conductivity. Note that $D_{\text {anion, NMR }}$ could not be measured directly from ${ }^{19} \mathrm{~F}$ tracer diffusion since the fluorine spin-spin relaxation time was found to be too short (more information available in SI). Since TTP is the only F-containing species, $D_{\text {anion,NMR }}$ was determined by measuring the diffusion of the TTP proton instead.

The room temperature ionic conductivities of LiTTP/diglyme are approximately three times higher than that of $\mathrm{LiOTf} /$ diglyme at all salt concentrations, reaching the values of $\sigma_{\mathrm{AC}}=3.3 \mathrm{mS} \mathrm{cm}^{-1}$ for $c_{\text {salt }}=0.45 \mathrm{M}$, which is another indication of improved dissociation of the lithium salt in LiTTP/diglyme (Figure 2b). Such conductivities are slightly higher than in typical 'salt-in-solvent' electrolytes at comparable $c_{\text {salt. }}{ }^{28}$

a

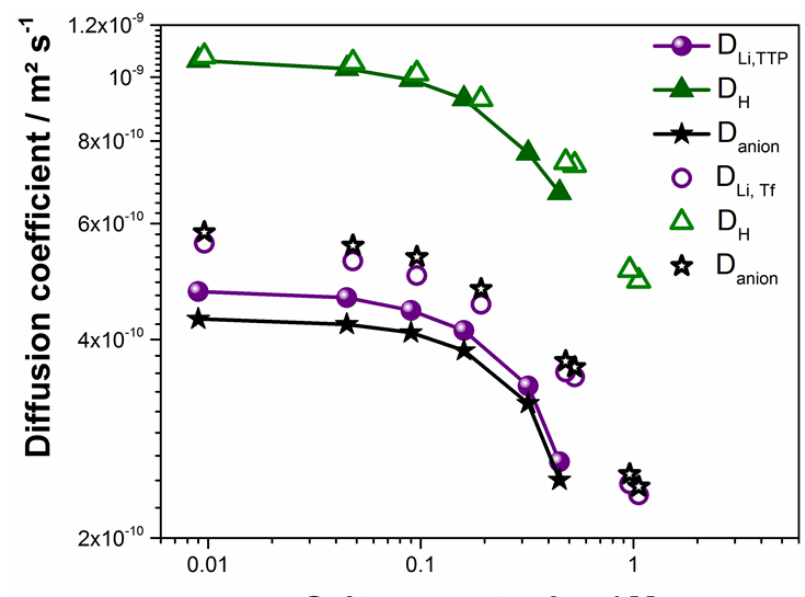

b

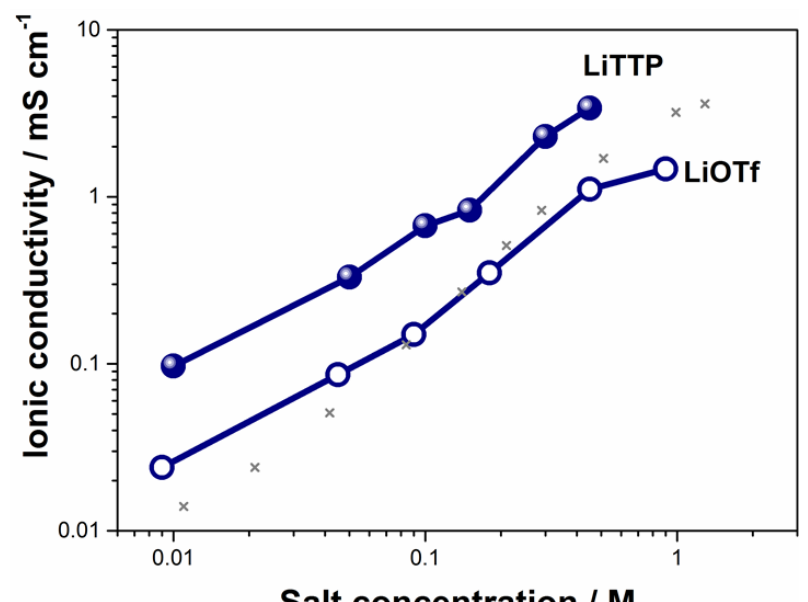

Figure 2. Lithium salt concentration dependent: (a) diffusion coefficients of species measured by PFG NMR, (b) ionic conductivities, $\sigma_{\mathrm{AC}}$, in diglyme electrolytes measured by impedance spectroscopy. LiTTP/diglyme electrolytes are marked with filled symbols, LiOTf/diglyme electrolytes with open symbols. Gray symbols in (b) correspond to the ionic conductivities of LiOTf/diglyme previously reported in Ref. 30 . The measurements were performed at room temperature.

The salting-out phenomenon occurs only at highest salt concentration $0.45 \mathrm{M}$, after a shelf time of several days, resulting in a crystalline 1:1 salt to diglyme mixture. This mixture has been isolated from the excess solvent and was used to measure $\mathrm{D}_{\mathrm{Li}}, \mathrm{NMR} \approx 2 \times \mathrm{D}_{\text {anion, }}, \mathrm{NMR}=9.8 \times 10^{-12} \mathrm{~m} 2 \mathrm{~s}-1$ and $\mathrm{D}_{\mathrm{H}}, \mathrm{NMR}=7.2 \times 10^{-12}$ at $100{ }^{\circ} \mathrm{C}$.

LiTTP/diglyme electrolytes can be polarized using low frequency impedance spectroscopy in a symmetric anionblocking lithium cell. ${ }^{28,29}$ Subsequently, the lithium transference number can be calculated independently of the solid electrolyte interphase (SEI) formation by taking into account the electrolyte resistance, $R_{\mathrm{b}}$, and the low frequency arc with diameter $Z_{d}$, via

$t_{L i}=\frac{1}{1+\frac{Z_{d}(0)}{R_{b}}}$,

Eq. 1

as depicted in Figure 3a. This low frequency impedance spectroscopy (ac) method is equivalent to the dc method proposed by Bruce and Evans, and could be converted to it by Fourier transformation. However, low frequency impedance spectroscopy allows for direct determination of the SEI contribution, while the SEI remains hidden in the current vs. time curve in the Bruce-Evans method, frequently not allowing for precise steady state determination. The obtained lithium transference numbers reach the highest value for LiTTP at $c_{\mathrm{salt}}=0.32 \mathrm{M}, t_{\mathrm{Li}}=0.7$, accompanied by high ionic conductivity, $\sigma_{\mathrm{AC}}=2.3 \mathrm{mS} \mathrm{cm}^{-1}$. The lithium transference number is slightly lower for $0.45 \mathrm{M} \mathrm{LiTTP} / \mathrm{diglyme}$, $t_{\mathrm{Li}}=0.6$. Precise determination of $t_{\mathrm{Li}}$ at lower salt concentrations, $c_{\text {salt }}<0.3 \mathrm{M}$, proved to be difficult, as the second semicircle becomes small and flattens out (Fig. S2). In addition to these measurement issues, the polarization needs to be performed as soon as the cell is assembled since LiTTP/diglyme electrolytes are not stable with the Li electrodes and quickly develop a highly resistive SEI (Fig. 3b and inset). The SEI instability of LiTTP/diglyme compared to other lithium glyme electrolytes is most probably a consequence of the changes in chemical composition of the SEI due to decomposition of the TTP anion, resulting in additional electronic conductivity or higher dimensional defects in the SEI. 
a

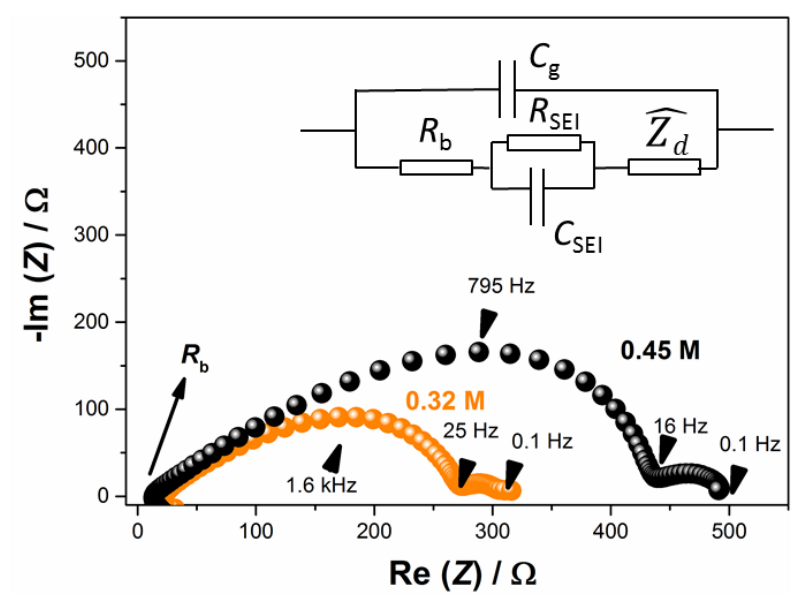

b

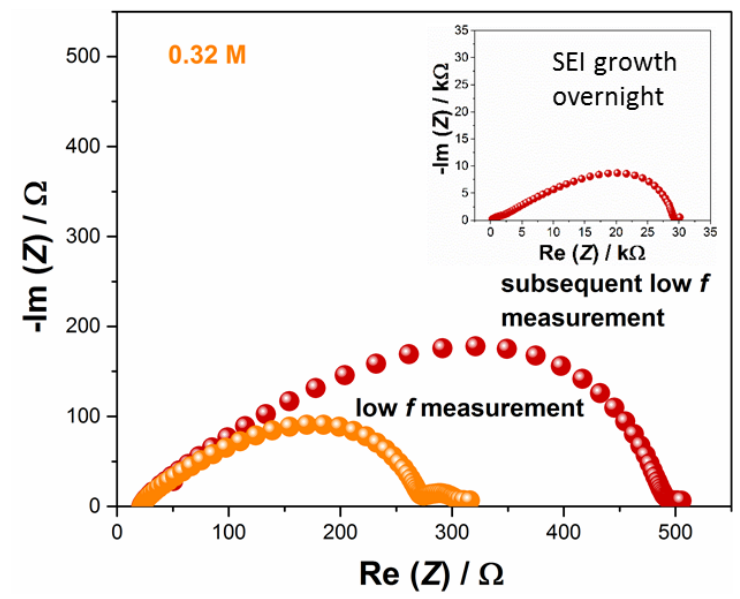

Figure 3. (a) Impedance spectroscopy of LiTTP/diglyme electrolytes in an anion blocking Li/electrolyte/Li cell: Determination of the lithium transference number by the low frequency method for $0.32 \mathrm{M}$ (orange) and $0.45 \mathrm{M}$ (black) LiTTP. The corresponding equivalent circuit is shown in the inset; b) solid electrolyte interphase (SEI) growth in $0.32 \mathrm{M}$ LiTTP during the low frequency ac-measurement. The inset shows highly resistive SEI forming overnight. The measurements were performed at room temperature.

The lithium conductivity originating from solvated free lithium ions, $\sigma_{+}$, can be linked to the diffusion coefficient through the Nernst-Einstein equation:

$\sigma_{+}=\frac{(z F)^{2} c_{+} D_{+}}{R T}$

Eq. 2

where $z$ is the ionic charge, $F$ is the Faraday constant, $R$ is the gas constant, $T$ is the temperature, $C_{+}$is the molar concentration of the free lithium ions, and $D_{+}$is the diffusion coefficient of the free lithium ions. In the present case, the direct determination of $\sigma_{+}$is unmanageable for two reasons: i) we do not have the access to the LiTTP/diglyme molecular structure and accordingly $c_{+}$as its determination by infrared or Raman spectroscopy is challenging and no

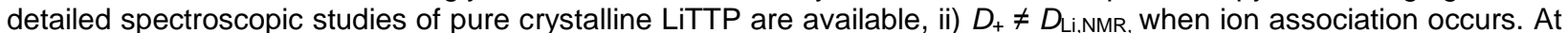
this point, we assume the existence of free ions and ion pairs only, while the exceptionally bulky dimers and higher order aggregates are neglected. The rationale behind such approximation lies in the fact that previous reports show insignificant conductivity contribution of even smaller dimers in LiOTf/diglyme and the molecular structure of TTP sterically does not allow for dimers. ${ }^{30,31}$

The concentration dependent transport coefficient of cations, $\sigma_{+}$, anions, $\sigma_{\text {-, }}$ and ion pairs, $s$, are accessible as solutions of a set of equations involving the total ionic conductivity, $\sigma_{\mathrm{AC}}$, and the conductivities of $\mathrm{Li}$ and $\mathrm{F}$-species calculated from the Nernst-Einstein equation for corresponding $D_{\mathrm{Li}}$ and $D_{\mathrm{F}}$, and total salt concentration $c_{\mathrm{s} a l t}, \sigma_{\mathrm{Tr}}^{L i}$ and $\sigma_{T r}^{F}$, as previously shown in Ref. 32:

$\sigma_{+}=\frac{\sigma_{A C}}{2}+\frac{\sigma_{T r}^{L i}}{2}-\frac{\sigma_{T r}^{F}}{2}$,

Eq. 3

$\sigma_{-}=\frac{\sigma_{A C}}{2}-\frac{\sigma_{T r}^{L i}}{2}+\frac{\sigma_{T r}^{F}}{2}$,

Eq. 4

$S=-\frac{\sigma_{A C}}{2}+\frac{\sigma_{T r}^{L i}}{2}+\frac{\sigma_{T r}^{F}}{2}$.

Eq. 5

Bruce and Vincent were the first to give a detailed account on the origin of the contributions of free ions and ion pairs and their role in the polarization experiment. ${ }^{34}$ The problem is isomorphic to the treatment of ions and electrons in solids where ion-electron association occurs, see Ref. 35 . 
The corresponding $c_{\text {salt }}$-dependent true lithium transference number, which takes into account lithium transport only via solvated free ions, can then be defined as $t_{+}=\frac{\sigma_{+}}{\sigma_{A C}}$, while the effective lithium transference number that also takes into account counter transport of ion pairs and anions, Ref. 32 as:

$$
t_{p o l,+}=\frac{\sigma_{p o l}}{\sigma_{A C}}=\frac{\sigma_{+}+\frac{\sigma_{-} s}{\sigma_{-}+s}}{\sigma_{A C}}
$$

It is important to note that the value of $t_{p o l,+}$ is more relevant for battery application since in a real cell we usually deal with partially blocking electrodes. In this way, even at $c_{\text {salt }}<0.3 \mathrm{M}$, where low frequency impedance spectroscopy fails, we can calculate all relevant transport coefficients.

In LiTTP/diglyme electrolyte, we observe for the first time in a liquid 'salt-in-solvent' electrolyte, that a situation in which $s>\sigma_{+}>\sigma_{\text {- }}$ for all accessible $c_{\text {salt }}$ is indeed realized (Figure 4a). The true lithium transference number is calculated to be $t_{+}>0.55$, with negligible $c_{\text {salt }}$ dependence. Contrary to LiOTf/diglyme, where $t_{p o l,+}$ is only slightly above 0.5 at very low $c_{\text {salt }}$ (Figure S3 and Ref. 31), calculated $t_{p o l,+}$ in LiTTP/diglyme are always above 0.8 , reaching unprecedentedly high values around 0.9 for $c_{\text {salt }}<0.2 \mathrm{M}$ (Figure $4 \mathrm{~b}$ ). Slight differences between the values calculated from Eq. 3-6 and the ones extracted from the low frequency impedance spectroscopy are either a consequence of imprecise determination of $R_{\mathrm{b}}$ and $Z_{\mathrm{d}}$ from the impedance spectra or due to the existence of a small amount of negatively charged higher order aggregates steering lithium in the undesirable direction. ${ }^{36,37}$ The observed drop of $s$ at higher $c_{\text {salt }}$, and accordingly $t_{+}$and $t_{p o l,+}$, is most probably caused by the rise in viscosity, which is in accordance with the Stokes - Einstein relationship, $D \propto 1 / \eta$. Indeed, the values of diffusion coefficients also rapidly drop in the same concentration range, see Figure $2 \mathrm{a}$.

a

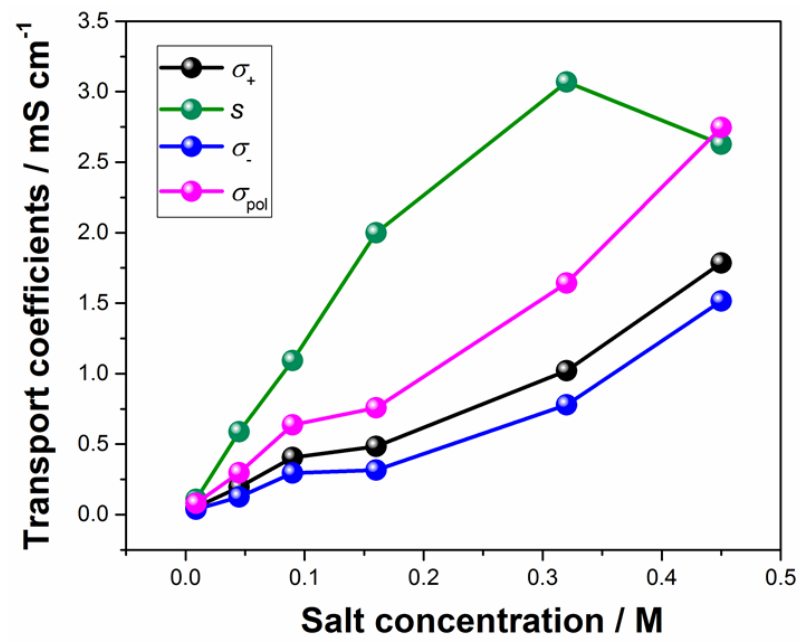

b

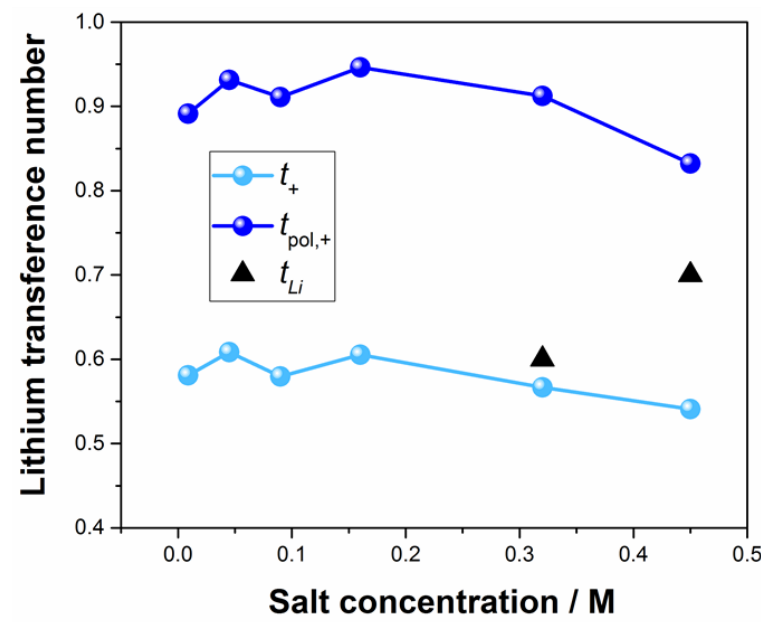

Figure 4. Calculated LiTTP salt concentration dependent: (a) transport coefficients of cations, $\sigma_{+}$, anions, $\sigma_{-}$, ion pairs, $s$, and indirect transport of lithium, $\sigma_{\mathrm{pol}}$, (b) effective lithium transference numbers that take into account lithium transport through ion pairs and solvated free lithium, $t_{\text {pol+ }}$, or only the transport by solvated lithium ions, $t_{+}$. The triangles in (b) correspond to the lithium transference numbers measured by a polarization experiment, $t_{\mathrm{L} i}$.

In conclusion, a 'salt-in-solvent' electrolyte containing the newly synthesized LiTTP salt displaying very high values of lithium transference numbers is reported. Polarization experiments yield transference numbers around $70 \%$ while ac impedance spectroscopy and PFG-NMR point towards even higher values and more complex ion-conduction mechanism.

The beneficial ionic conductivity of LiTTP/diglyme as compared with the LiOTf/diglyme electrolyte is a consequence of the interplay between reduced mobilities of large solvated anions and lithium transport through smaller amount of ion pairs. Future studies will be directed towards the stabilization of the Li metal/electrolyte interfaces. 


\section{ASSOCIATED CONTENT}

Supporting Information. Synthesis and characterization of LiTTP; details on preparation of electrolytes, pulse field gradient NMR and impedance spectroscopy measurements

\section{AUTHOR INFORMATION}

The authors declare no competing financial interests.

\section{ACKNOWLEDGEMENTS}

The authors thank Florian Kaiser, Uwe Traub, Udo Klock, and Petra Wedemann for technical support. Generous support by the Max-Planck-Society, the Deutsche Forschungsgemeinschaft (Leibniz Award to B.L. and Cluster of Excellence RESOLV, EXC 1069), and the European Research Council (Advanced Grant "C-H Acids for Organic Synthesis, CHAOS") is gratefully acknowledged.

\section{REFERENCES}

(1) Henderson W. A. in Electrolytes for Lithium and Lithium-Ion Batteries (Eds.: T. R. Jow, K. Xu, O. Borodin, M. Ue), Springer New York, New York, NY 2014

(2) Younesi, R.; Veith, G. M.; Johansson, P.; Edström, K.; Vegge, T. Lithium Salts for Advanced Lithium Batteries: Li-Metal, Li-O and Li-S, Energy Environ. Sci. 2015, 8, 1905.

(3) Xu, K. Nonaqueous Liquid Electrolytes for Lithium-Based Rechargeable Batteries, Chem. Rev. 2004, 104 (10), 4303.

(4) Bogle, X; Vazquez, R.; Greenbaum, S.; Cresce, A. v. W.; Xu, K. Understanding Li ${ }^{+}-$Solvent Interaction in Nonaqueous Carbonate Electrolytes with ${ }^{17}$ O NMR, J. Phys. Chem. Lett. 2013, 4(16), 1664.

(5) Giorgini, M. G.; Futamatagawa K; Torii, H.; Musso, M.; Cerini, S. Solvation Structure Around the Li ${ }^{+}$Ion in Mixed Cyclic/Linear Carbonate Solutions Unveiled by the Raman Noncoincidence Effect, J. Phys. Chem. Lett. 2015, 6(16), 3296.

(6) Chapman, N.; Borodin, O.; Yoon, T.; Nguyen, C. C.; Lucht, B. L. Spectroscopic and Density Functional Theory Characterization of Common Lithium Salt Solvates in Carbonate Electrolytes for Lithium Batteries, J. Phys. Chem. C 2017, 121 (4), 2135.

(7) Ong, M. T.; Bhatia, H.; Gyulassy, A. G.; Draeger, E. W.; Pascucci, V.; Bremer, P.-T.; Lordi, V.; Pask, J. E. Complex lon Dynamics in Carbonate Lithium-lon Battery Electrolytes, J. Phys. Chem. C 2017, 121(12), 6589.

(8) Liang, C.; Kwak, K.; Cho, M. Revealing the Solvation Structure and Dynamics of Carbonate Electrolytes in Lithium-lon Batteries by Two-Dimensional Infrared Spectrum Modeling, J. Phys. Chem. Lett. 2017, 8, 5779.

(9) Jonnson, E.; Armand, M.; Johansson, P. Novel Pseudo-Delocalized Anions for Lithium Battery Electrolytes, Phys. Chem. Chem. Phys. 2012, 14, 6021.

(10) Jankowski, P.; Wieczorek, W.; Johansson, P. New Boron Based Salts for Lithium-lon Batteries Using Conjugated Ligands, Phys. Chem. Chem. Phys. 2016, 18, 16274

(11) Krause, L. J.; Lamanna, W.; Summerfield, J.; Engle, M.; Korba, G; Loch, R.; Atanasoski, R. Corrosion of Aluminum at High Voltages in Non-Aqueous Electrolytes Containing Perfluoroalkylsulfonyl Imides; New Lithium Salts for Lithium-lon Cells, J. Power Sources 1997, 68(2), 320.

(12) H.-B.; Zhou, S.-S; Zhang, D.-J.; Feng, S.-F.; Li, L.-F.; Liu K.; Feng, W.-F.; Nie, J.; Li, H.; Huang, X.-J.; Armand, M.; Zhou, Z.-B. Lithium Bis(fluorosulfonyl)imide (LiFSI) as Conducting Salt for Nonaqueous Liquid Electrolytes for Lithium-lon Batteries: Physicochemical and Electrochemical Properties, J. Power Sources 2011, 196(7), 3623.

(13) Xu, W.; Angel, C. A. Weakly Coordinating Anions, and the Exceptional Conductivity of Their Nonaqueous Solutions, Electrochem. Solid-State Lett. 2001, 4(1), E1.

(14) Xu, W.; Schusterman, A. J.; Marzke, R.; Angell, C. A. LiMOB, an Unsymmetrical Nonaromatic Orthoborate Salt for Nonaqueous Solution Electrochemical Applications, J. Electrochem. Soc. 2004, 151(4), A632.

(15) Niedzicki, L.; Zukowska, G. Z.; Bukowska, M.; Szeczecinski, P.; Grugeon; Laruelle, S.; Armand, M.; Panero, S.; Scrosati, B.; Marcinek, M.; Wieczorek, W. New Type of Imidazole Based Salts Designed Specifically for Lithium Ion Batteries, Electrochim. Acta 2010, 55(4), 1450.

(16) Shkorb, I.; Pupek, K. Z.; Gilbert J. A.; Trask, S. E.; Abraham, D. P. Chemical Stability of Lithium 2-Trifluoromethyl-4,5dicyanoimidazolide, an Electrolyte Salt for Li-lon Cells, J. Phys. Chem. C 2016, 120 (50), 28463.

(17) Liao, C.; Han, K. S.; Baggetto, L.; Hillesheim, D. A.; Custelcean, R.; Lee, E.-S.; Guo, B.; Bi, Z.; Jiang, D.; Veith, G. M.; Hagaman, E. W.; Brown, G. B.; Bridges, C.; Paranthaman, M. P.; Manthiram, A.; Dai, S.; Sun, X.-G. Synthesis and Characterization of Lithium Bis(fluoromalonato)borate for Lithium-Ion Battery Applications, Adv. Energy Mater. 2014, 4(6), 1301368.

(18) Sund, X.-G.; Wan, S.; Guang, H. Y.; Fang, Y.; Reeves, K. S.; Chi, M.; Dai, S. New Promising Lithium Malonatoborate Salts for High Voltage Lithium Ion Batteries, J. Mat. Chem. A 2017, 5, 1233.

(19) Thiam, A.; lojoiu, C.; Leprêtre, J.-C.; Sanchez, J.-Y. Lithium Salts Based on a Series of New Anilinyl-Perfluorosulfonamide Salts and Their Polymer Electrolytes, J. Power Sources 2017, 361, 138.

(20) Shyamsunder, A.; Beichel, W.; Klose, P.; Pang, Q.; Scherer, H.; Hoffmann, A.; Murphy, G. K.; Krossing, I.; Nazar, L. Inhibiting Polysulfide Shuttle in Lithium-Sulfur Batteries through Low-lon-Pairing Salts and a Triflamide Solvent, Angew. Chem. Int. Ed. 2017, 56(11), 6192.

(21) Höfler, D.; van Gemmeren, M.; Wedemann, P.; Kaupmees, K.; Leito, I.; Leutzsch, M.; Lingnau, J. B.; List., B 1,1,3,3Tetratriflylpropene (TTP): A Strong, Allylic C-H Acid for Brønsted and Lewis Acid Catalysis, Angew. Chem. Int. Ed. 2016, 56(5), 1411.

(22) Davies, C. W. Ion Association, Butterworths: London, 1964.

(23) Barthel, G.; Krinke, H; Kunz. W. Physical Chemistry of Electrolyte Solutions, Springer, New York, 1998.

(24) Xu, K. Electrolytes and Interphases in Li-lon Batteries and Beyond, Chem. Rev. 2014, 114

(25) Price, W. S. NMR Studies of Translational Motion, University Press: Cambridge, 2009.

(26) Hayamizu, K.; Aihara, Y.; Arai, S.; Garcia Martinez, C. Pulse-Gradient Spin-Echo ${ }^{1} \mathrm{H}$, ${ }^{7} \mathrm{Li}$, and ${ }^{19} \mathrm{~F}$ NMR Diffusion and Ionic Conductivity Measurements of 14 Organic Electrolytes Containing LiN( $\left.\mathrm{SO}_{2} \mathrm{CF}_{3}\right)_{2}$, J. Phys. Chem. B 1999, 103(3), 519.

(27) Hayamizu, K.; Sugimoto, K.; Akiba, E.; Aihara, Y.; Bando, T.; Price, W. S. An NMR and lonic Conductivity Study of lon Dynamics in Liquid Poly (ethylene oxide)-Based Electrolytes Doped with LiN(SO $\left(\mathrm{SF}_{3}\right)_{2}$, J. Phys. Chem. B 2002, $106(3), 547$. 
(28) Gores, H. J.; Barthel, J.; Zugmann, S.; Moosbauer, D.; Amereller, M; Hartl, R.; Maurer, A. Liquid Nonaqueos Electrolytes in Handbook of Battery Materials (Eds: Daniel C., Besenhard, J. O.), Wiley-VCH, Weinheim, 2011.

(29) Sorensen, P. R.; Jacobsen, T. Conductivity, Charge Transfer and Transport Number-an ac-Investigation of the Polymer Electrolyte LiSCN-poly (ethyleneoxide), Electrochim. Acta 1982, 27(12), 1671.

(30) Petrowsky, M.; Frech, R.; Suarez, S. N.; Jayakody, J. R. P.; Greenbaum, S. Investigation of Fundamental Transport Properties and Thermodynamics in Diglyme-Salt Solutions, J. Phys. Chem. B, 2006, 110(46), 23012.

(31) Popovic, J.; Pfaffenhuber, C.; Melchior, J.; Maier, J. Determination of Individual Contributions to the lonic Conduction in Liquid Electrolytes: Case Study of LiTf/PEGDME-150 Electrochem. Comm., 2015, 60, 195.

(32) Maier, J. Concentration Polarization of Salt-Containing Liquid Electrolytes, Adv. Funct. Mat., 2011, $21,1448$.

(34) Bruce, P.; Vincent, C. A. Effect of Ion Association on Transport in Polymer Electrolytes, Faraday Discuss. Chem. Soc., 1989, $88,43$.

(35) Maier, J., Physical Chemistry of lonic Materials, John Wiley \& Sons, England, 2004.

(36) Maier, J., Salt Concentration Polarization of Liquid Electrolytes and Determination of Transport Properties of Cations, Anions, Ion Pairs and Ion Triples Electrochim. Acta, 2014, 129, 21.

(37) Gouverneur, M.; Schmidt, F.; Schönhoff, M. Negative Effective Li Transference Numbers in Li Salt/lonic Liquid Mixtures: Does Li Drift in the "Wrong" Direction?, Phys. Chem. Chem. Phys., 2018, 20, 7470. 